\title{
Micro-beam XRF and Fe-K Edge XAFS on the Cross Section of the Rust Layer Formed on a Weathering Steel
}

\author{
Masayasu NAGOSHI, ${ }^{1)}$ Takashi KAWANO, ${ }^{1)}$ Isamu KAGE ${ }^{1)}$ and Shinjiro HAYAKAWA ${ }^{2)}$ \\ 1) Steel Research Laboratory, JFE Steel Corporation, 1-1 Minami-watarida, Kawasaki 210-0855 Japan. E-mail: m-nagoshi@ \\ jfe-steel.co.jp $\quad$ 2) Graduate School of Engineering, Hiroshima University, Higashi-Hiroshima, Hiroshima $739-8527$ Japan.
}

(Received on July 22, 2010; accepted on August 30, 2010)

\begin{abstract}
Micro-beam X-ray fluorescence (XRF) and X-ray absorption fine structure (XAFS) techniques have been applied, for the first time, to the cross sections of a rust layer on a weathering steel exposed for 38 years to the atmospheric environment. Elemental mapping and Fe-K edge XAFS spectra were measured with a spatial resolution of about $3 \mu \mathrm{m}$ by an X-ray micro beam formed by a K-B mirror with the synchrotron radiation. $\mathrm{Cr}$ distribution, including layered patterns in the rust layer, was clearly observed by the micro-beam XRF imaging. The combination of micro-XRF and extended X-ray absorption fine structure (EXAFS) analysis on identical analytical points has demonstrated that the crystallinity of $\alpha$-FeOOH is strongly related to the $\mathrm{Cr}$ concentration. The XRF and XAFS results are presented and discussed in terms of the analytical techniques and the relationship between the crystal structure of the rust and the $\mathrm{Cr}$ concentration.
\end{abstract}

KEY WORDS: XAFS; XRF; EXAFS; micro-beam; micro-analysis; synchrotron radiation; rust; weathering steel; corrosion; $\mathrm{Cr}$; scale; $\mathrm{FeOOH}$.

\section{Introduction}

Weathering steel with small amounts of alloying elements such as $\mathrm{P}, \mathrm{Cr}$, and $\mathrm{Cu}$ has been widely used for structural steel products, bridges, buildings, and other steel constructions, because the corrosion rates of the steel are significantly lower compared to the ordinary carbon steel in atmospheric corrosion environments. ${ }^{1)}$ Dense rust layers which form on the steel surface (inner rust layer) with the enrichments of alloying elements mentioned above have been considered to act as a protective layer against the penetration of aggressive corrosive anions such as $\mathrm{Cl}^{-}$into the steel surfaces. Therefore, the structures of the inner rust layer have been extensively studied. ${ }^{2-11)}$

Various advanced techniques, such as Raman spectroscopy, ${ }^{2-4)}$ transmission electron microscopy (TEM), ${ }^{5-7)}$ and X-ray absorption fine structure (XAFS) ${ }^{6-11)}$ have been applied to reveal the nanostructure of the rust layer in addition to basic optical microscopy (OM), X-ray diffraction (XRD), and electron-probe microanalysis (EPMA) techniques. XAFS has played a very important role in understanding the nanostructures of corrosion products. Especially, this technique is very powerful for investigating chemical states and local structures of added elements in low concentrations and atomic-scale positions of $\mathrm{Cr}$ and $\mathrm{Ni}$ in corrosion products have been proposed. Although $\mathrm{Fe}-\mathrm{K}$ edge XAFS analysis has the potential to reveal nanometerscale structures of the corrosion products directly, there are the following problems if we apply the techniques to the rust layer formed on weathering steels.

Few Fe-K XAFS measurements have been carried out on bulk surfaces covered by rust layers ${ }^{6,7)}$ or on powdered specimens scraped off from corroded steel surfaces. ${ }^{10)}$ These approaches have provided averaged information on the rust layer. It should be noted that the rust layers of the weathering steel usually consist of double-layered structures as indicated by OM and EPMA. ${ }^{2)}$ Moreover, the inner rust layers which are important for corrosion resistance layers are not uniform but have complex distributions of alloyed elements and crystal structures including amorphous phases, as shown by EPMA, ${ }^{2)}$ Raman spectroscopy, ${ }^{4)}$ and $\mathrm{TEM}^{5-7)}$ data for the rust on weathering steels exposed for more than 10 years. The Fe-K edge XAFS data measured for the powder specimens or bulk specimens have provided little information concerning to these in-homogeneity. In order to discuss the microscopic structure of inner rust and the formation mechanisms of the layer by XAFS, the data from well-characterized micron-level positions in the rust layer are needed.

We have applied micro-beam XRF and XAFS techniques using synchrotron radiation, for the first time, to the cross section of a rust layer formed on a weathering steel. The performance of this approach to clarify the local structures of an in-homogeneous rust layer was examined. The distribution of elements especially $\mathrm{Cr}$ and the nanostructure of the corrosion products are investigated for the rust formed on a weathering steel exposed for long time in an atmospheric environment. The results are compared with the previous studies. 


\section{Experimental}

\subsection{Specimen}

A piece of weathering steel exposed to atmospheric environment for 38 years at a seaside industrial area in Japan was used for this study. Table 1 shows the chemical composition of this steel. The surface of the specimen was covered by black or dark brown corrosion products. The X-ray diffraction pattern of the rust powder revealed that the main compound in the rust is $\alpha-\mathrm{FeOOH}$ and as well as a small amount of $\gamma$-FeOOH exists as shown in Fig. 1. A large fraction of the rust layer covering the steel surface showed dark contrast in cross sectional optical microscopic images observed by reflected polarized light (a datum is shown in Fig. 3(a)). By comparing these characteristics with results in the previous reports, ${ }^{2)}$ it is concluded that the surface of the weathering steel used in this study is covered by similar protective corrosion products.

The cross section of the specimen was prepared by polishing using ethanol after having been immersed in a resin for micro-beam XRF and XAFS. The specimens were set as face to face in the resin in order to prevent the rust layer from being broken off during polishing treatments. It was confirmed through optical microscopic observation that the cross section surface of the rust layer was flat.

\subsection{Micro-beam XRF and XAFS}

Micro-beam XRF and XAFS measurements were carried out under atmospheric conditions at the beamline BL37XU, SPring-8, Hyogo, Japan. The micro-beam X-ray was generated by a pair of $\mathrm{Rh}$ coated elliptical mirrors (Kirkpatrick and Baez mirror, $\mathrm{K}-\mathrm{B}$ mirror) inserted into the optical path. The central glancing angle of the mirror was $4 \mathrm{mrad}$, and $\mathrm{X}$-rays up to $20 \mathrm{keV}$ could be focused. The intensity of the incident X-ray was monitored by an ionization chamber. The details of the experimental systems used in this study were reported in Ref. 12).

The beam size of the focused X-ray beam of $10 \mathrm{keV}$ was evaluated by an edge-scan of a gold wire of $50 \mu \mathrm{m}$ in diameter. The wire mounted on the specimen holder was moved across the focused beam, and the transmitted X-rays were

Table 1. Chemical composition of the steel used in this study (mass\%).

\begin{tabular}{ccccccc}
\hline $\mathrm{C}$ & $\mathrm{Si}$ & $\mathrm{Mn}$ & $\mathrm{P}$ & $\mathrm{Cu}$ & $\mathrm{Cr}$ & $\mathrm{Ni}$ \\
\hline 0.046 & 0.14 & 0.38 & 0.049 & 0.27 & 0.84 & 0.18 \\
\hline
\end{tabular}

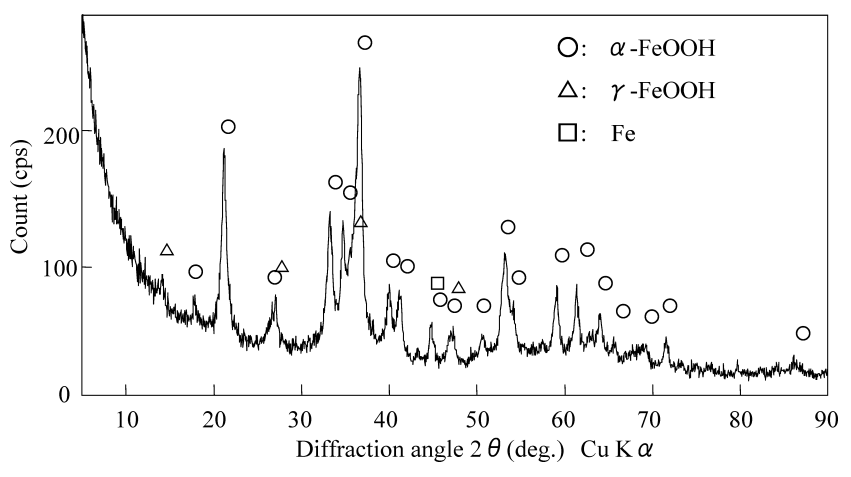

Fig. 1. X-Ray diffraction pattern with the $\mathrm{Cu}-\mathrm{K} \alpha$ line for the rust powder scraped off from the specimen. monitored by the ionization chamber. Figure 2 shows the profiles of the edge-scans and their derivatives. The beam size of the focused incident X-ray was estimated to be less than $3 \mu \mathrm{m}$ (FWHM) in the horizontal and vertical directions, and the beam size was conserved within the tunable energy range.

XAFS spectra were measured by the X-ray fluorescence yield mode by using a Si drift detector (SDD). The detector was set in the electron orbit plane of the storage ring and mounted at 90 degrees to the incident X-ray. The detector was placed $130 \mathrm{~mm}$ apart from the beam position on the sample, and an aperture of $4 \mathrm{~mm}$ in diameter was placed in front of the detector to restrict the solid angle. The specimen was placed as the angle between the polished specimen surface (cross section of the rust layer) and the SDD was 2 degrees (the incident angle was 88 degrees from the specimen surface) to reduce the self-absorption effects in XAFS spectra. ${ }^{13)}$ A XAFS spectrum of a bulk steel surface measured by this experimental system was compared with that of an iron foil measured by the transmission mode. The obtained peak heights in Fourier transform (FT) were about $85 \%$ of those with the reference iron foil. The result indicates that the self-absorption effect was not completely removed in our experimental setup. However, the relative intensities of the peaks from the first shell to the fifth shell were almost identical between the two data. This implies that we can compare relative peak intensities in the FTs between the micro-beam XAFS data and for reference materials obtained by the transmission XAFS experiments. The XRF experiments were also carried out using the same ex-
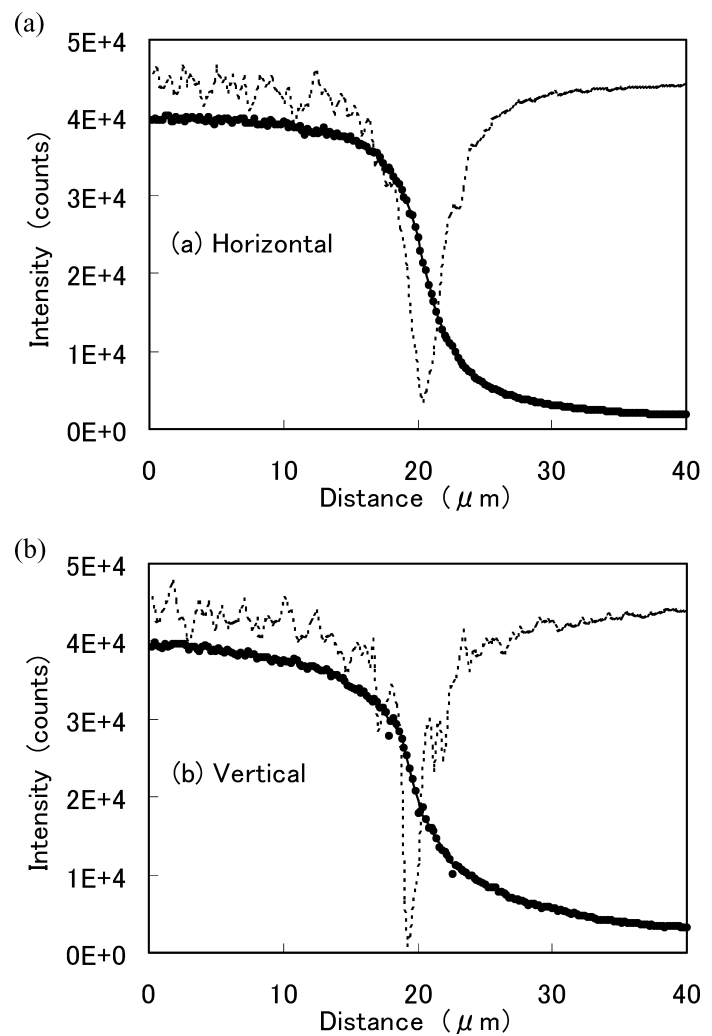

Fig. 2. Profiles of edge-scans and derived beam profiles obtained with the focused X-ray beam of $10 \mathrm{keV}$. Raw data (dots) were smoothed with the Savitzky-Golay 11 point method (solid line) and their derivatives-represent the beam profiles in horizontal and vertical directions. 
perimental geometry as mentioned for the XAFS experiments.

\subsection{Other Experiments}

Fe-K edge XAFS spectra for the rust powder scraped off from the same piece of the specimen and commercial $\alpha$ $\mathrm{FeOOH}$ and $\gamma$-FeOOH powders were measured on the transmission mode at beam line BL-27, Photon Factory, KEK, Tsukuba. These specimens were ground and mixed with $\mathrm{BN}$ powder and then the mixtures were formed into pellets. The beam size of the incident X-ray was set to $4 \mathrm{~mm} \times 1 \mathrm{~mm}$ in order to obtain averaged information of the powdered rust. There are no essential differences on the transmission-mode XAFS spectra between two experimental systems used in this study for homogeneous specimens. Actually, the equivalent XAFS spectra were obtained for an iron foil on the transmission mode at both beam lines.

After the micro-beam X-ray experiments, the distribution of elementals was measured by an electron probe micro analyzer (EPMA), JXA-8900, JEOL on the identical crosssectional surface after deposition with evaporated carbon. The accelerating voltage and the beam current of the incident electrons were set to $15 \mathrm{kV}$ and $1 \times 10^{-7} \mathrm{~A}$, respectively. This experimental condition provides spatial resolution of about $1 \mu \mathrm{m}$.

\section{Results and Discussion}

\subsection{XRF Mapping by Micro-beam X-ray}

Figure 3(a) shows the cross sectional image of the rust layer observed by an optical microscope with a polarized light. A large fraction of the rust layer shows a dark color (dark area in Fig. 3(a)) and some parts show a bright orange color (bright area in Fig. 3(a)). The former has been considered to the stable corrosion products consisting of $\alpha$ $\mathrm{FeOOH}$ and the latter has been considered to $\gamma-\mathrm{FeOOH}$ according to the previous reports. ${ }^{2)}$

The distribution of the X-ray fluorescence intensity ratio of $\mathrm{Cr}-\mathrm{K} \alpha$ to $\mathrm{Fe}-\mathrm{K} \alpha$ is shown in Fig. 3(b), in which areas with higher $\mathrm{Cr}-\mathrm{K} \alpha$ relative intensity are denoted by the brighter contrast. Enrichment and depletion of $\mathrm{Cr}$ in the rust layer are clearly seen, when we compare the brightness with those on the steel region. Double-layered structures of the rust are clearly seen in part B where $\mathrm{Cr}$ is enriched with a layered distribution in the inner layer and is depleted in the outer layer. By a comparison of the $\mathrm{Cr}-\mathrm{K} \alpha / \mathrm{Fe}-\mathrm{K} \alpha$ intensity ratio with the optical image in Fig. 3(a), it is found that the areas with $\mathrm{Cr}$-enrichment correspond to the areas where dark contrast was observed in the polarized optical image.

The roughness of the sample surface could affect fluorescence X-ray intensities due to the shallow detection angle used in this study. Actually, discontinuous changes of both $\mathrm{Cr}-\mathrm{K} \alpha$ and $\mathrm{Fe}-\mathrm{K} \alpha \mathrm{X}$-ray intensity were observed at the interface between the steel and the rust layer where slight steps were introduced by the polishing treatment. These changes of X-ray intensities, however, almost all disappear by taking $\mathrm{X}$-ray intensity ratio, $\mathrm{Cr}-\mathrm{K} \alpha / \mathrm{Fe}-\mathrm{K} \alpha$, as shown in Fig. 3(b).

The distribution of $\mathrm{Cr}-\mathrm{K}$ intensity was measured by EPMA after the micro-beam experiments. Figure 3(c)
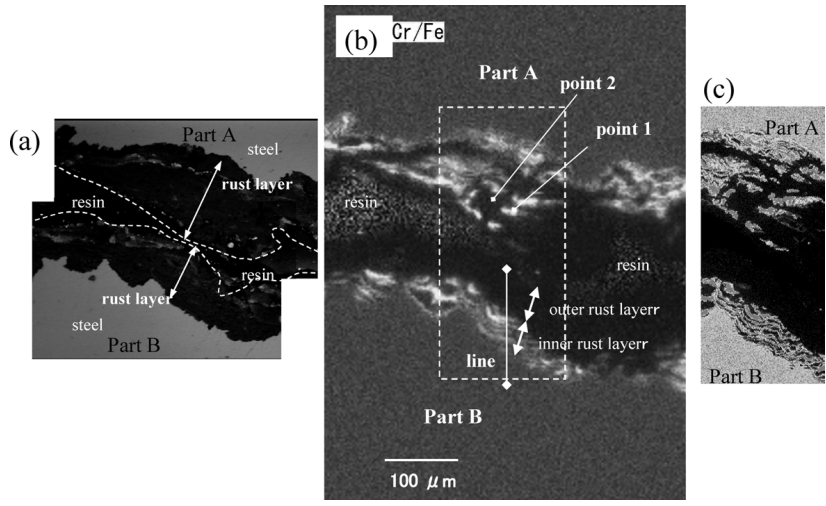

Fig. 3. Optical microscopic image with polarized light (a), XRF intensity ratio image $\mathrm{Cr}-\mathrm{K} \alpha / \mathrm{Fe}-\mathrm{K} \alpha$ measured by microbeam XRF with an energy of $7.8 \mathrm{keV}$ (b), and $\mathrm{Cr}-\mathrm{K} \alpha \mathrm{X}$ ray intensity mapping measured by EPMA (c) for the cross section of the weathering steel exposed for 38 years. The images in (a), (b), and (c) were obtained from the identical area. EPMA mapping area is denoted by a dashed rectangle in (b). Points and line where XRF and XAFS spectra were recorded are denoted in figure (b).

shows the $\mathrm{Cr}-\mathrm{K}$ intensity mapping image corresponding to the delineated area in the center of Fig. 3(b), in which areas with higher $\mathrm{Cr}-\mathrm{K}$ intensity are denoted by the brighter contrast. The $\mathrm{Cr}-\mathrm{K} \alpha / \mathrm{Fe}-\mathrm{K} \alpha$ intensity ratio mapping measured by micro-beam XRF is in good agreement with the $\mathrm{Cr}-\mathrm{K} \alpha$ intensity mapping obtained by EPMA. Although the spatial resolution of the XRF mapping is rather poorer compared to the Cr mapping by EPMA (the spatial resolution is about $1 \mu \mathrm{m})$, almost all $\mathrm{Cr}$ enriched areas with a width of more than $2-3 \mu \mathrm{m}$ on the EPMA mapping were detected by the micro-beam XRF. This demonstrates that the spatial resolution of about $3 \mu \mathrm{m}$ is achieved by this micro-beam XRF system for the cross section of the rust layer. The incident angle of X-rays close to the normal surface ( 88 degrees) limits the spread of the projection area of the incident X-ray on the sample surface. The shallow detection angle (2 degrees) limited the escape depth of $\mathrm{Fe}-\mathrm{K} \alpha \mathrm{X}$-ray to about $1 \mu \mathrm{m}$. The detection geometry used in this study helps the conservation of lateral spatial resolution.

The cross section of the specimen was observed by the optical microscope before and after the micro-beam XRF and XAFS experiments. No significant changes were observed in the images even when the observation was done with polarized light. This suggests that no damage was caused in the cross sectional surface regions by the microbeam X-ray irradiation. This would be an advantage of micro-beam X-ray techniques compared to electron beam techniques (SEM, EPMA, TEM) in vacuum conditions and laser techniques such as Raman spectroscopy. Actually, cracks were introduced on the cross section of the rust by the EPMA measurements. The XAFS focuses on the target elements, which is an advantage when compared with Raman spectroscopy, a vibration spectroscopy.

\subsection{Micro-beam XAFS}

Figure 4(a) shows enlarged XRF spectra for points 1 and 2 (the positions are denoted in Fig. 3(b)) where $\mathrm{Cr}$ is enriched and depleted, respectively. These data clearly show 

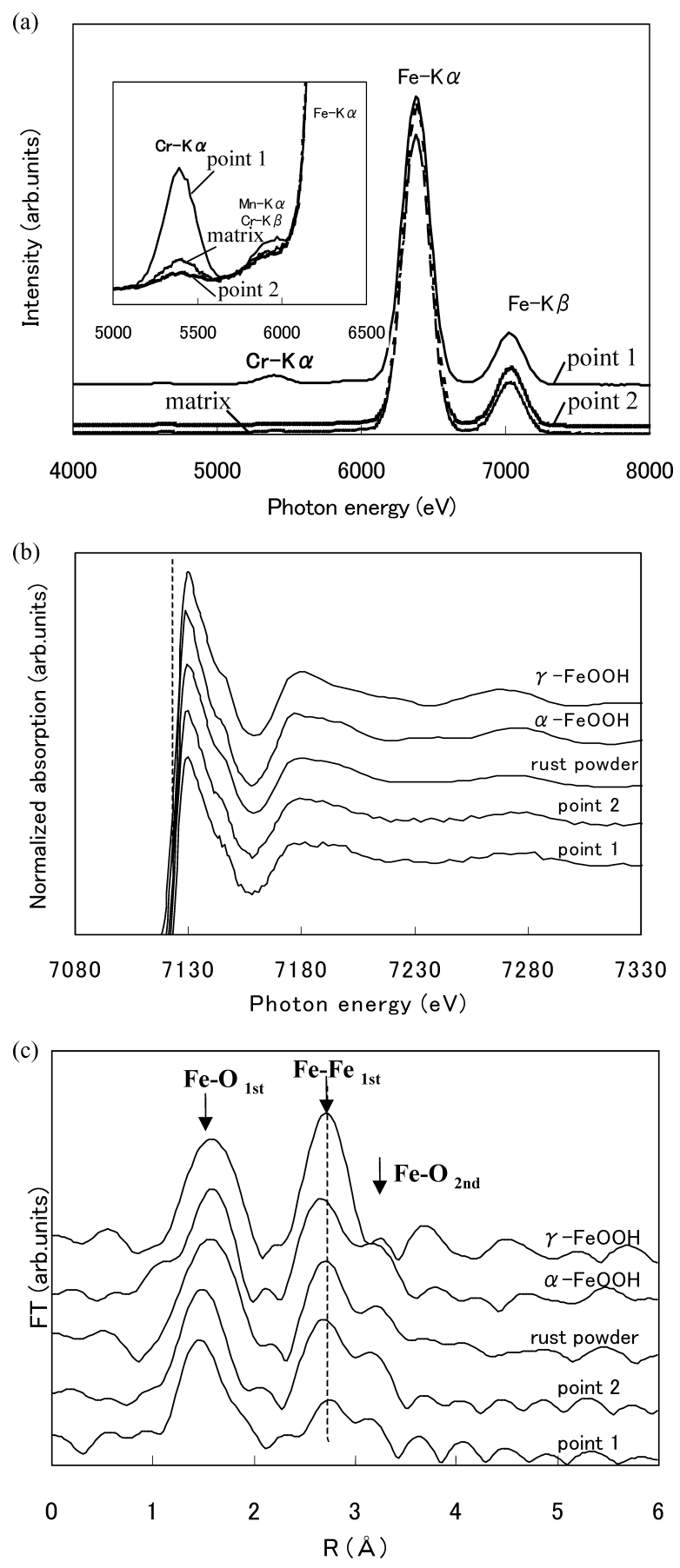

Fig. 4. Micro-beam XRF spectra (a), normalized XAFS spectra (b), and FTs (c) for the points 1 and 2 shown in Fig. 3(b) on the cross-section of the rust layer. The data for the rust powder scraped off from the specimen surface and commercial $\alpha$-FeOOH and $\gamma$-FeOOH are shown in Figs. 4 (b) and 4(c). The FTs in (c) are normalized in intensity as the peaks at about $1.5 \AA$ have the same height.

the difference of $\mathrm{Cr}$ concentration between the two points. The net intensity ratio of the $\mathrm{Cr}-\mathrm{K} \alpha$ peak to the $\mathrm{Fe}-\mathrm{K} \alpha$ peak was estimated to about 5 times (point 1) and about 0.4 times (point 2) of that for the steel region.

XAFS spectra obtained for the points 1 and 2 are shown in Fig. 4(b) with spectra for the rust powder scraped off from the surface of the specimen and for commercial materials; $\alpha$-FeOOH and $\gamma$-FeOOH. X-Ray absorption nearedge structure (XANES) spectra for the rust specimens in Fig. 4(b) are more similar to that for $\alpha$-FeOOH rather than that for $\gamma$-FeOOH. This result shows that the chemical states of the main corrosion products are present at the analytical point close to that of $\alpha-\mathrm{FeOOH}$. By comparing the spectra at the photon energies from 7150 to $7350 \mathrm{eV}$, the spectral shape for point 2 is close to that for the rust powder. The spectral shape for point 1 is slightly different from those for point 2 and the rust powder. This suggests that there are some modifications in the $\alpha-\mathrm{FeOOH}$ structure at point 1 where $\mathrm{Cr}$ is enriched.

Figure 4(c) shows the Fourie transform (FT) derived from $k^{3}$-weighted EXAFS oscillations in the $k$ range of 3-11 (1/A) for points 1 and 2. The data for the rust powder specimen and the commercial $\alpha-\mathrm{FeOOH}$ and $\gamma-\mathrm{FeOOH}$ are also shown. The FT corresponds to the radial distribution function (RDF) obtained by XAFS in which the horizontal axis is not corrected by the phase shift. Peaks at about $1.5 \AA$ are attributed to the nearest neighboring oxygen atoms around $\mathrm{Fe}$ atoms. The second and third peaks at about $2.7 \AA$ and $3.2 \AA$ have been attributed to the mixture of the first neighboring $\mathrm{Fe}$ atom groups and the second neighboring oxygen atom groups. $\left.{ }^{6}\right)$ The peak shapes of the second and third peaks for the points 1 and 2 are similar to each other and are also similar to those for $\alpha-\mathrm{FeOOH}$. However the relative heights of the second and third peaks normalized to the first peak are significantly different; the peak heights for point 1 with enriched $\mathrm{Cr}$ are lower than that for point 2 with depleted $\mathrm{Cr}$. The crystal structure of $\alpha$-FeOOH consists of ordered edge-sharing and point-sharing of $\mathrm{Fe}-(\mathrm{O}, \mathrm{OH})_{6}$ octahedrons. We denote this characteristic as the short-range ordering of $\alpha$-FeOOH in this paper. The lower peak height for the first neighboring $\mathrm{Fe}$ atoms and second neighboring oxygen atoms (but the relative intensity between them is not changed) indicates that the short-range ordering of the Fe$(\mathrm{O}, \mathrm{OH})_{6}$ network of $\alpha$-FeOOH is more distorted in the $\mathrm{Cr}$ enriched rust than in the $\mathrm{Cr}$-depleted rust.

In Fig. 4(c), the peak heights of the second and third peaks for the rust powder are the same level as those in point 2. This result can be explained through the fact that the Cr-depletion rust has a larger volume fraction in the rust layer, with the consequence that the information from the Cr-enriched rust is diluted by the Cr-depletion rust. The mixing with information from $\gamma-\mathrm{FeOOH}$ is also a possible reason. This evidently shows that it is not possible to estimate accurately the effects of $\mathrm{Cr}$ on the local structures of corrosion products from $\mathrm{Fe}-\mathrm{K}$ edge XAFS data obtained for rust powders scraped off from rusted steel surfaces.

The micro-beam XRF and XAFS approach was applied to the Cr-enriched rust with the layered distributions. Figure 5(a) shows a line profile of the $\mathrm{Cr}-\mathrm{K} \alpha / \mathrm{Fe}-\mathrm{K} \alpha$ intensity ratio (the intensities of background are included in each intensity) obtained by the micro-beam XRF measurements as a function of the distance along the line indicated at part $\mathrm{B}$ in Fig. 3(b). It is clearly found that $\mathrm{Cr}$ is enriched in the inner rust layer with intensity variation and is depleted at the outer rust layer. The FT obtained by the micro-beam XAFS for the selected points denoted in Fig. 5(a) are shown in Fig. 5(b). In this figure, the vertical scales of FT were normalized as that the peaks of the first nearest neighboring oxygen atoms at about $1.5 \AA$ have the same height except for the FT for point 1.

The FT for the rust layer in Fig. 5(b) can be divided to 

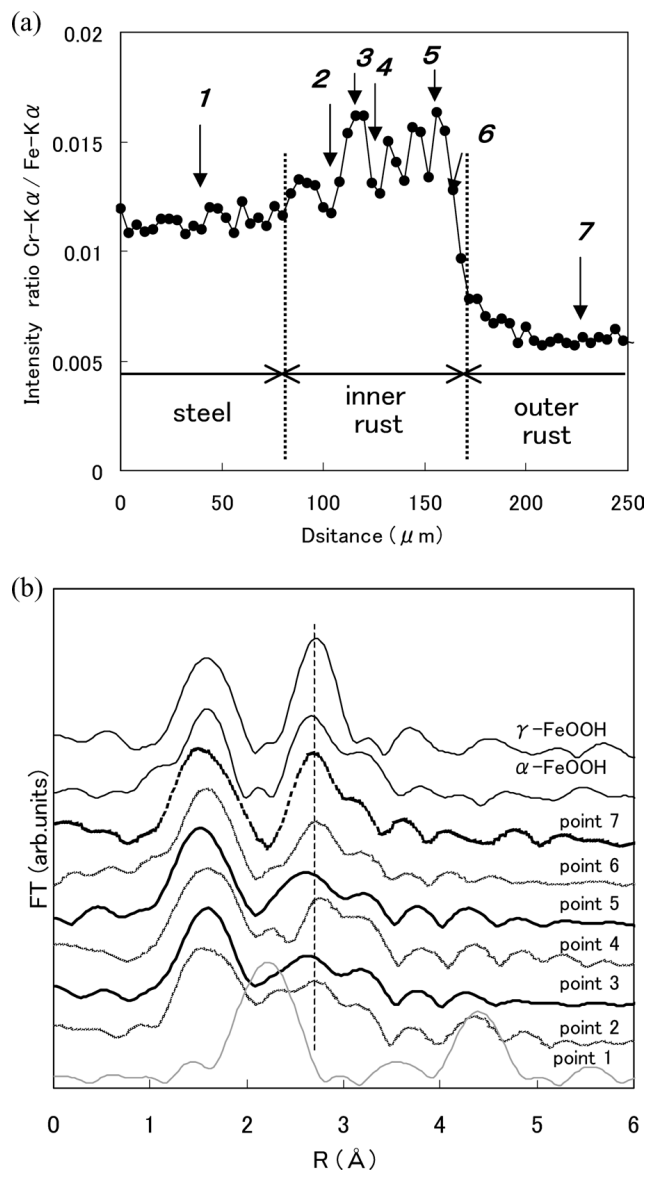

Fig. 5. Line profile (a) of $\mathrm{Cr}-\mathrm{K} \alpha / \mathrm{Fe}-\mathrm{K} \alpha$ intensity ratio measured along the line shown in Fig. 3(b). FTs (b) for selected points in Fig. (a). The FTs are normalized in intensity as the peaks at about $1.5 \AA$ have the same height.

three classes by the heights of the second and third FT peaks at $2.5-3.5 \AA$ although the peak shapes for all points are similar to those for $\alpha$-FeOOH rather than those for $\gamma$ $\mathrm{FeOOH}$. The FT for $\mathrm{Cr}$-enriched points at the inner rust layer, points 3 and 5 , have the lowest peak heights among the points 2-7. The FTs for the point in which $\mathrm{Cr}-\mathrm{K} \alpha / \mathrm{Fe}-$ $\mathrm{K} \alpha$ intensity ratios are relatively low at the inner rust layer, points 2, 4, and 6, have medium peak heights. Point 7 in the outer rust layer with the lowest $\mathrm{Cr}-\mathrm{K} \alpha / \mathrm{Fe}-\mathrm{K} \alpha$ intensity ratios shows the FT with the highest peak height.

The relative heights of the second peak at about $2.7 \AA$ in the FT are plotted in Fig. 6 as a function of the $\mathrm{Cr}-\mathrm{K} \alpha$ relative intensity obtained by XRF for the identical points shown in Fig. 5. The $\mathrm{Cr}-\mathrm{K} \alpha$ relative intensities, $\mathrm{Cr}-\mathrm{K} \alpha / \mathrm{Fe}-$ $\mathrm{K} \alpha$, were normalized to that for the steel region (point 1). The $\mathrm{Cr}-\mathrm{K} \alpha / \mathrm{Fe}-\mathrm{K} \alpha$ ratios are calculated after subtracting the background intensity for $\mathrm{Cr}-\mathrm{K} \alpha$ and $\mathrm{Fe}-\mathrm{K} \alpha$ peaks in the XRF spectra measured by micro-beam X-ray with the energy of $8 \mathrm{kV}$ for each points. There is a good inversely linear correlation between the intensity of the second peak in the FT and the relative $\mathrm{Cr}-\mathrm{K} \alpha$ intensity. For other alloying elements $\mathrm{Cu}$ and $\mathrm{Ni}$, broad distributions in the inner rust layer and the lack of a sharp layered-structure seen for $\mathrm{Cr}$ were observed by micro-XRF imaging. These results strongly suggest that $\mathrm{Cr}$ plays a direct role on controlling of nano-scale ordering of $\alpha$-FeOOH crystals during crystal formation and/or aging of the corrosion products.

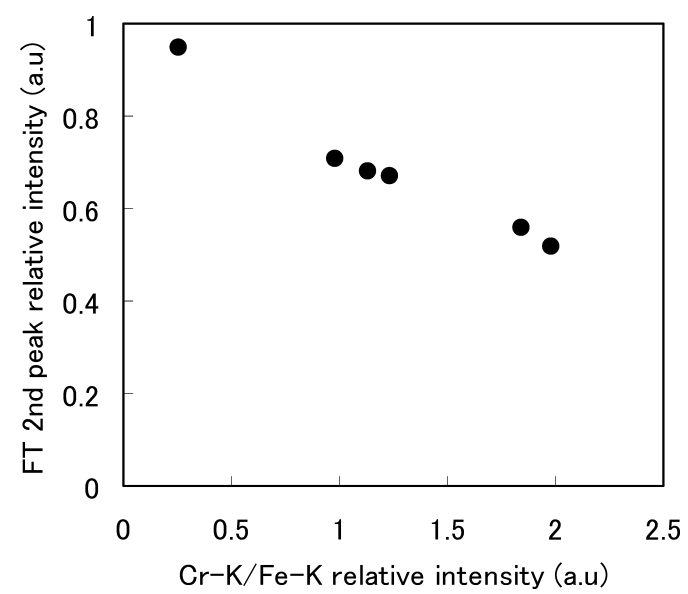

Fig. 6. Relationship between $\mathrm{Cr}-\mathrm{K} \alpha / \mathrm{Fe}-\mathrm{K} \alpha$ intensity ratio normalized to the value for the steel substrate and relative peak intensity of 2 nd peak at about $2.7 \AA$ in FT diagrams normalized to that of the peak at about $1.5 \AA$ for the analysis point in Fig. 5.

It was reported that the chromium makes $\alpha$-FeOOH grains small ${ }^{3,4)}$ or poorly crystalline through investigation of laboratory-synthesized $\mathrm{FeOOH}$ with additions of $\mathrm{Cr}$ ions into solutions. Microscopic mechanisms of $\mathrm{Cr}$ on forming fine $\alpha$-FeOOH crystals have been proposed from $\mathrm{Cr}-\mathrm{K}$ edge XAFS analysis. ${ }^{6-11)}$ For the rust layer on weathering steels it has been reported that the rust with higher $\mathrm{Cr}$ concentration corresponds to $\alpha$-FeOOH with smaller sized or more disordered structures as revealed by Raman spectroscopy ${ }^{4)}$ and TEM. ${ }^{6,7)}$ Our micro-XRF and XAFS results reveal the relationship between $\mathrm{Cr}$ concentration and the disorder of $\alpha$ $\mathrm{FeOOH}$ in the rust on the weathering steel after atmospheric exposure for 38 years from the view point of the short-range order around iron atoms in the $\alpha$-FeOOH structure. The previous Raman study ${ }^{4)}$ showed that $\mathrm{Cr}$ concentration gradually increases from the surface of the inner layer to the rust/steel interface. This tendency is different from our result, as we observed a more complex distribution of $\mathrm{Cr}$ concentration and the disorder of $\alpha$-FeOOH. The Raman measurements were carried out with intervals of 3-24 $\mu \mathrm{m}$, which is a possible reason for this difference because the limited number of analytical points will smear out in-homogeneity with a size of less than few micrometers.

The spatial resolution higher than about $3 \mu \mathrm{m}$ which was obtained in this study is desired because the order of in-homogeneity of the rust layer is often smaller than this value. Recently, the progress of micro-probe X-ray techniques with the synchrotron radiation have allowed smaller-size Xray beams. ${ }^{14,15)}$ It is expected that the X-ray with the beam size of less than sub-micron level will focus on nanometerlevel structures of more complex corrosion products and reveal mechanisms of corrosion-resistance by trace elements.

\section{Conclusions}

Micro-beam XRF and XAFS have been applied to the cross section of the rust layer formed on the weathering steel exposed to atmospheric environment for 38 years. The main results are as follows.

(1) The micro-beam XRF and XRF-yield XAFS meas- 
urements with a shallow detection angle in air have successfully provided elemental mappings and pinpoint $\mathrm{Fe}-\mathrm{K} \alpha$ XAFS spectra used for EXAFS analysis with a spatial resolution of about $3 \mu \mathrm{m}$ on the cross section of a rust layer. The characteristics of the in-homogenous rust layer revealed in this study are impossible to obtain by ordinary XAFS experiments for powder rust specimens and for surfaces covered by a rust layer. The micro-beam XRF and XAFS have an advantage of lower damage to the rust surfaces compared with the analytical techniques with electron or laser beams.

(2) The $\mathrm{Cr}$ distributions including layered patterns in the rust layer were clearly observed by the micro-beam XRF imaging. It was found by the combination of the micro-beam XAFS and XRF measurements of the identical points on the cross section that the short-range ordering of $\alpha$-FeOOH crystals is distorted in the Cr-enriched rust compared to the Cr-depleted rust.

(3) There is a good inversely linear correlation between the short-range ordering of $\alpha$-FeOOH crystals derived from the micro-beam XAFS and the relative concentration of $\mathrm{Cr}$ estimated by the micro-beam XRF. This result strongly suggests that the $\mathrm{Cr}$ plays a direct role in nanometer-scale orderings of the $\alpha$-FeOOH crystal in the crystal formation and/or crystal growth processes during exposure to a corrosion environment for a long time.

\section{Acknowledgement}

The micro-beam XRF and XAFS experiments were carried out as the SPring-8 Experimental Proposal No. 2006A1441 "Cross-sectional structural analysis for rust layer of steel studied by micro-beam XAFS". We would like to thank JASRI for their support with the experiments.
Two of the present authors (N.M and T.K) thank the High Energy Acceleration Research Organization (KEK) and Prof. K.Kobayashi, KEK, for their fruitful collaboration at Photon Factory.

\section{REFERENCES}

1) For example, I. Matsushima: Low-Alloy Corrosion Resistant Steel, Chijin Shokan, Tokyo, (1995).

2) T. Misawa, M. Yamashita, Y. Matsuda, H. Miyuki and H. Nagano: Tetu-to-Hagané, 79 (1992), 69.

3) M. Yamashita, H. Miyuki, Y. Matsuda, H. Nagano and T. Misawa: Corrosion Sci., 36 (1994), 283.

4) M. Yamashita, H. Miyuki, H. Nagano and T. Misawa: Tetu-toHagané, 83 (1997), 36.

5) K. Shiotani, W. Tanioto, C. Maeda, F. Kawabata and K. Amano: Zairyou-to-Kankyou, 49 (2000), 67.

6) M. Kimura, T. Suzuki, G. Shigesato, H. Kihira and S. Suzuki: ISIJ Int., 42 (2002), 1534.

7) M. Kimura, T. Suzuki, G. Shigesato, H. Kihira and S. Suzuki: Surf Interface Anal., 35 (2003), 66.

8) M. Yamashita, H. Uchida, H. Konishi and J. Mizuki: Mater. Trans., 45 (2004), 1920.

9) H. Konishi, J. Mizuki, M. Yamashita and H. Uchida: Mater. Trans., 46 (2005), 136.

10) H. Konishi, J. Mizuki, M. Yamashita and H. Uchida; Mater. Trans., 46 (2005), 329.

11) H. Konishi, J. Mizuki, M. Yamashita and H. Uchida: Mater. Trans., 46 (2005), 337.

12) S. Hayakawa, N. Ikuta, M. Suzuki, M. Wakatsuki and T. Hirokawa: $J$. Synchr. Radiat., 8 (2001), 328.

13) S. Hayakawa, Y. Gohshi, A. Iida and K. Sato: Rev. Sci. Instrum., 62 (1991), 2545.

14) Y. Suzuki, A. Takeuchi, H. Takano and H. Talenala: Jpn. J. Appl. Phys., 44 (2005), 1994.

15) S. Matsuyama, H. Miura, H. Yumoto, Y. Sano, K. Yamamura, M. Yabashi, Y. Nishino, K. Tamasaku, T. Ishikawa and K. Yamauchi: Rev. Sci. Instrum., 77 (2007), 103102. 